\title{
$\beta$-catenin blocks Kras-dependent reprogramming of acini into pancreatic cancer precursor lesions in mice
}

\author{
John P. Morris IV, David A. Cano, Shigeki Sekine, Sam C. Wang, and Matthias Hebrok
}

Diabetes Center, Department of Medicine, UCSF, San Francisco, California, USA.

\begin{abstract}
Cellular plasticity in adult organs is involved in both regeneration and carcinogenesis. WT mouse acinar cells rapidly regenerate following injury that mimics acute pancreatitis, a process characterized by transient reactivation of pathways involved in embryonic pancreatic development. In contrast, such injury promotes the development of pancreatic ductal adenocarcinoma (PDA) precursor lesions in mice expressing a constitutively active form of the GTPase, Kras, in the exocrine pancreas. The molecular environment that mediates acinar regeneration versus the development of PDA precursor lesions is poorly understood. Here, we used genetically engineered mice to demonstrate that mutant Kras promotes acinar-to-ductal metaplasia (ADM) and pancreatic cancer precursor lesion formation by blocking acinar regeneration following acute pancreatitis. Our results indicate that $\beta$-catenin is required for efficient acinar regeneration. In addition, canonical $\beta$-catenin signaling, a pathway known to regulate embryonic acinar development, is activated following acute pancreatitis. This regeneration-associated activation of $\beta$-catenin signaling was not observed during the initiation of Kras-induced acinar-to-ductal reprogramming. Furthermore, stabilized $\beta$-catenin signaling antagonized the ability of Kras to reprogram acini into PDA preneoplastic precursors. Therefore, these results suggest that $\beta$-catenin signaling is a critical determinant of acinar plasticity and that it is inhibited during Kras-induced fate decisions that specify PDA precursors, highlighting the importance of temporal regulation of embryonic signaling pathways in the development of neoplastic cell fates.
\end{abstract}

\section{Introduction}

Pancreatic ductal adenocarcinoma (PDA) is the fourth leading cause of cancer death in the United States (1). Analysis of patient samples and genetically engineered mouse models suggests that it likely develops from preneoplastic ductal lesions, including pancreatic intraepithelial neoplasias (PanINs) (2). Understanding how signaling pathways interact in the pancreatic epithelium to elicit PanINs therefore represents a key step in the possible development of tools for early PDA detection and treatment. While PanINs express markers of ductal differentiation, many recent studies suggest that they can arise from pancreatic acinar cells that are reprogrammed into a preneoplastic state by undergoing acinar-toductal metaplasia (ADM) (3-7).

Activating mutations in the gene encoding the GTPase Kras are nearly universal in human PDA (8), and targeting of mutated Kras to mouse pancreatic progenitors recapitulates the human PanIN-toPDA progression sequence (9). While ADM is observed in these models (6) and Kras can spontaneously induce ADM and PanIN formation when activated in adult acini $(3,4,7)$, expression of mutant Kras in acinar cells does not guarantee ductal reprogramming. Acini expressing mutant Kras during embryogenesis appear grossly unaffected (9), and some normal acinar tissue is maintained in the context of PanIN and PDA progression as mice age $(9,10)$. Therefore, additional events must occur to enable a cellular environment that permits Kras to drive ADM/PanIN formation.

Recently, environmental insults that result in exocrine damage and inflammation have been shown to represent one such permissive environment. For example, Guerra and colleagues (5) and Car-

Conflict of interest: The authors have declared that no conflict of interest exists. Citation for this article: J Clin Invest. 2010;120(2):508-520. doi:10.1172/JCI40045. riere and colleagues (11) have demonstrated that chronic and acute chemical pancreatitis, respectively, induced by the cholecystokinin receptor agonist caerulein accelerate development of ADM/PanIN and PDA progression when mutant Kras is expressed in the acinar/ centroacinar compartment. These findings, particularly of Guerra and colleagues (5), support clinical data showing that chronic pancreatitis represents a potent risk factor for PDA $(12,13)$. However, the exact manner by which active Kras coopts exocrine damage to initiate the PanIN to PDA sequence is unclear.

In response to caerulein-induced acute pancreatitis, WT mouse acinar cells decrease expression of acinar markers and reactivate factors expressed during pancreatic development $(14,15)$. These cells may also assume a transient duct-like morphology $(16,17)$. Lineage tracing has revealed that it is mainly preexisting acini, rather than a dedicated acinar stem cell, that rapidly redifferentiate and repopulate the acinar compartment following caerulein pancreatitis (16, 17). Thus, this transient dedifferentiation of acini into duct-like cells during regeneration differs from $\mathrm{ADM}$, in which ductal differentiation becomes fixed. Nonetheless, acini undergoing ADM both in vitro due to EGF ligand signaling $(18,19)$ and in vivo in the context of mutant Kras share expression of some embryonic markers of regenerating WT acinar cells, such as Nestin and Notch pathway activation $(14,16)$. Since expression of many of these reactivated embryonic factors is also maintained throughout PanIN and PDA development, such as Notch signaling (20), achieving a less-differentiated expression profile that resembles pancreatic development may play a role in PanIN initiation. Furthermore, some factors reactivated during acinar regeneration have also been shown to promote ADM. For example, while the transcription factor pancreatic and duodenal homeobox 1 (Pdx1), which itself is critical to embryonic pancreatic development, is transiently upregulated dur- 
ing acinar regeneration (14), enforced exocrine expression induces ADM (21). This suggests that reactivation of developmental signaling pathways must be tightly regulated to permit acinar regeneration but prevent persistent ductal reprogramming.

Given the ability of mutant Kras to spontaneously induce ADM and PanINs, which share molecular similarity to regenerating acini, we set out to investigate the effects of mutant Kras on differentiation and regeneration-associated signaling pathways immediately following acinar damage. Using acute caerulein pancreatitis as a model, we found that mutant Kras blocks acinar regeneration and promotes persistently dedifferentiated $\mathrm{ADM} /$ PanIN. Probing for the molecular mechanisms underlying this switch, we found that regeneration-associated $\beta$-catenin signaling was inhibited during Kras-induced acinar-to-ductal reprogramming and that $\beta$-catenin was required for efficient acinar regeneration. Finally, we show that stabilized $\beta$-catenin signaling antagonizes Kras-induced ADM and prevents the formation of acinar-derived PanINs. Thus, we conclude that $\beta$-catenin signaling is a key modulator of acinar plasticity and represents a critical molecular difference between acinar regeneration and Krasinduced reprogramming of acini into PDA precursors.

\section{Results}

Mutant Kras blocks acinar regeneration in favor of ADM/PanIN formation. To determine how mutant Kras affects acinar regeneration, we generated mice in which a constitutively active form of Kras, LSL-Kras ${ }^{G 12 D}$ (where LSL indicates lox site, transcriptional stop, lox site) (9), is induced via Cre recombinase expressed in all pancreatic progenitors by the $P d x 1$ promoter $P d x 1$-Cre $e^{E a r l y}(22)$. At 6 weeks of age, the pancreas in these mice comprises mostly morphologically normal acini with few ductal PanIN lesions despite efficient recombination at the conditional Kras locus (Figure 1, E and M). We induced acute caerulein pancreatitis using the "staggered" protocol described in Methods and compared regeneration in control Pdx1-Cre ${ }^{\text {Early }}$ and Pdx1-Cre ${ }^{\text {Early }} ; L S L-K r a s^{G 12 D}$ mice. The exocrine compartments of both cohorts responded comparably 2 days after caerulein treatment, displaying degranulated acinar cells (Figure 1, B and F), decreased amylase expression (Figure 1, J and N), and duct-like structures consistent with acute pancreatitis (Figure 1, $\mathrm{B}, \mathrm{F}$, and insets). These structures were morphologically similar to the transient ductal intermediates reported by other groups during WT acinar regeneration $(16,17)$. In Pdx 1 -Cre $e^{\text {Early }}$ control mice, these structures also weakly coexpressed the duct marker CK19, while CK19 was strongly expressed in comparable structures in $P d x 1$ Cre ${ }^{E a r l y} ; L S L-K r^{G 12 D}$ animals (compare Figure 1, J, N, and insets). By 7 days following caerulein treatment, regeneration of the acinar compartment of Pdx1-Cre Early control animals was nearly complete, as amylase and CK19 expression was redistributed in a fashion similar to that seen with PBS treatment (Figure 1, C and K). Similarly, pancreata at 21 days following caerulein treatment were indistinguishable from those of PBS-treated animals (Figure 1, D and L).

In stark contrast, acinar regeneration was blocked in $P d x 1-C r e^{\text {Early; }}$; $L S L-$ Kras $^{G 12 D}$ mice, resulting in replacement of the normal exocrine compartment with ductal structures. Seven days following treatment, the parenchyma of Pdx1-Cre Early; LSL-Kras ${ }^{G 12 D}$ pancreata was mainly replaced by CK19-positive duct structures embedded in an expanded stromal compartment (Figure 1, G and $\mathrm{O}$ ). Morphologically normal amylase-expressing acinar cells were rare, with some amylase-positive cells found in duct structures (Figure 1O). By 21 days following treatment, the exocrine compartment was predominately composed of CK19-positive, mucinous ductal lesions resembling low-grade PanINs (Figure 1, $\mathrm{H}$ and $\mathrm{P}$ ). As observed in other reports of spontaneous mouse PanINs (9), these lesions frequently stained for Alcian blue, a marker of mucin accumulation in human PanINs (Supplemental Figure 1B; supplemental material available online with this article; doi:10.1172/JCI40045DS1). Consistent with histological features at both time points and a progression in severity of the observed defects, Alcian blue-positive structures were more common at day 21 following caerulein treatment than 7 days after treatment (compare Supplemental Figure 1, A and B).

To further determine whether acinar reprogramming is a source of the abnormal ducts and PanINs resulting from inhibited acinar regeneration, we also performed this analysis using transgenic mice that have Kras activation in a more restricted set of cells during embryogenesis $\left(P d x 1-C r e^{L a t e} ; L S L-K_{r a s}{ }^{G 12 D}\right)$ as well as mice in which Kras activity is restricted to adult acinar cells (Elastase-Cre ${ }^{E R T 2}$;LSLKras $\left.^{G 12 D}\right)$. We have previously shown that in the $P d x 1$-Cre ${ }^{\text {Late }}$ driver line, Cre activity is found in acinar cells but notably absent from ducts (22). Caerulein treatment in these mice displayed a time course of subverted regeneration and accelerated ADM/PanIN development similar to that in Pdx1-Cre ${ }^{E a r l y} ; L S L-K r a s^{G 12 D}$ mice (Supplemental Figure 1, C-F), supporting acinar reprogramming as a source of the observed duct structures and PanINs.

To more rigorously determine whether acini serve as the cell of origin for ADM/PanIN following inhibited acinar regeneration, we also performed these experiments with Elastase-Cre ${ }^{E R T 2}$-driven mouse models. This line was chosen, as it permits tamoxifen-inducible Cre activation restricted to adult acini (23). To trace cells in which Cre was active, we also included a $R 26 R$-EYFP reporter line in our matings, which possesses a Cre-inducible lox-stop-lox-EYFP cassette targeted to the Rosa26 locus (24). Elastase-Cre ${ }^{E R T 2} ; R 26 R$ EYFP and Elastase-Cre ${ }^{E R T 2} ; L S L-K r a s^{G 12 D} ; R 26 R-E Y F P$ mice were generated and stimulated with tamoxifen (see Methods) at 4 weeks of age. Two weeks later, mice were treated with PBS or the staggered caerulein protocol as described above. As expected, yellow fluorescent protein (YFP) expression was limited to amylase-positive acinar cells and absent from CK19-positive duct cells in tamoxifen-stimulated, PBS-treated Elastase-Cre ${ }^{\text {ERT2}}$;R26R-YFP mice (Supplemental Figure $1 \mathrm{G})$. Two days following caerulein treatment, however, we observed double-CK19/YFP-positive cells in duct-like structures (Supplemental Figure 1H) similar to those observed in Pdx1-Cre $e^{E a r l y}$ control mice (Figure 1, F and J), supporting a transient ductal state during acinar regeneration. These double-CK19/YFP-positive cells may be similar to the acinar-derived type 1 tubular complexes (TC1) duct-like structures described by Strobel and colleagues (17). They also resemble double-amylase/CK19-positive structures observed during regeneration following partial pancreatectomy in rats (25). Of note, we also found CK19-positive acinar structures that were YFP negative. Due to the mosaic nature of CreERT2 activation, we cannot rule out that these are cells in which YFP was not activated. Alternatively, they may represent nonacinar-derived TC2 structures, as described previously (17). As expected, upon regeneration 7 and 21 days after caerulein treatment, YFP expression was again limited to acinar cells (Supplemental Figure 1, I and J).

Tamoxifen-stimulated Elastase-Cre ${ }^{E R T 2} ; L S L-K r a s^{G 12 D}$ and Elastase$C r e^{E R T 2} ; L S L-K r a s^{G 12 D} ; R 26 R-E Y F P$ mice treated with PBS possessed grossly normal pancreata (Supplemental Figure $1 \mathrm{~K}$ ) that rarely demonstrated ADM/PanIN (1 of 6 mice; 4 to 5 weeks following tamoxifen). Except for rare lesions in Elastase-Cre ${ }^{E R T 2} ; L S L$ - 

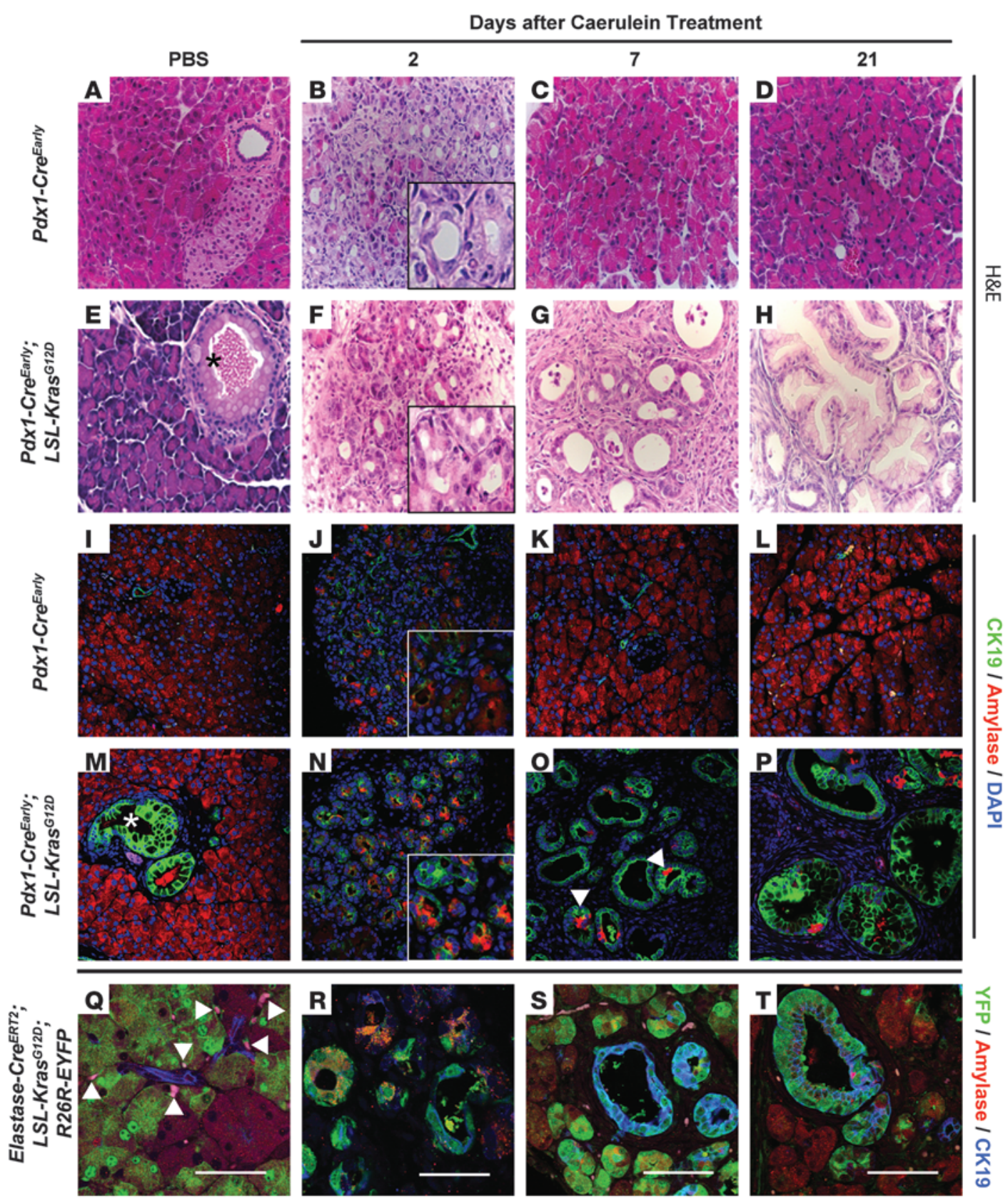

Figure 1

Mutant Kras blocks acinar regeneration and promotes ADM/PanIN formation. (A-H) H\&E staining of regeneration time course in Pdx1-CreEarly $(\mathbf{A}-\mathbf{D})$ and Pdx1-Cre Early; LSL-Kras G12D (E-H) mice. (E and M) Asterisks indicate spontaneous PanIN lesions in PBS-treated Pdx1-CreEarly;LSLKras ${ }^{G 12 D}$ animals. Insets in $\mathbf{B}$ and $\mathbf{F}$ show morphologically similar duct-like cells 2 days after induction of acute pancreatitis. (I-P) Amylase (red)/ CK19 (green) immunofluorescent labeling. Note CK19 expression in spontaneous PanIN lesions in Pdx1-Cre ${ }^{\text {Early; }}$ LSL-Kras ${ }^{G 12 D}$ mice (asterisk, $\mathbf{M})$. ( $\mathbf{J}$ and $\mathbf{N}$ ) Amylase is downregulated and CK19 is weakly expressed in transient duct-like cells in Pdx1-CreEarly mice (inset, $\mathbf{J}$ ) while strongly expressed in duct-like cells in Pdx1-Cre Early;LSL-Kras G12D mice (inset, N). Rare amylase-positive cells are found in metaplastic epithelium (arrowheads, O). (Q-T) Amylase (red), CK19 (blue), YFP (green) immunofluorescent labeling in Elastase-Cre ${ }^{E R T} ; L S L-K r a S^{G 12 D} ; R 26 R-E Y F P$ mice. Without caerulein treatment, YFP expression is restricted to amylase-positive cells and restricted from CK19-positive cells. Arrowheads indicate autofluorescent erythrocytes (Q). Double-CK19/YFP-positive cells (cyan, indicating blue/green overlap) persist following caerulein treatment $(\mathbf{R}-\mathbf{T})$. Original magnification, $\times 400(\mathbf{A}-\mathbf{P}$; insets). Scale bars: $50 \mu \mathrm{m}(\mathbf{Q}-\mathbf{T})$.

$\operatorname{Kras}^{G 12 D} ; R 26 R-E Y F P$ mice, YFP expression was limited to acinar cells and excluded from CK19-positive ducts (Figure 1Q). Two days following caerulein treatment, Elastase-Cre $e^{E R T 2} ; L S L$ Kras $^{G 12 D}$;R26R-EYFP mice developed duct-like structures similar to those observed in other genotypes (Supplemental Figure 1L), which were composed of acinar-derived, double-CK19/YFP-positive cells (Figure 1R). In contrast to Elastase-Cre ${ }^{E R T 2} ; R 26 R-E Y F P$ control mice, acinar-derived double-CK19/YFP-positive ducts 


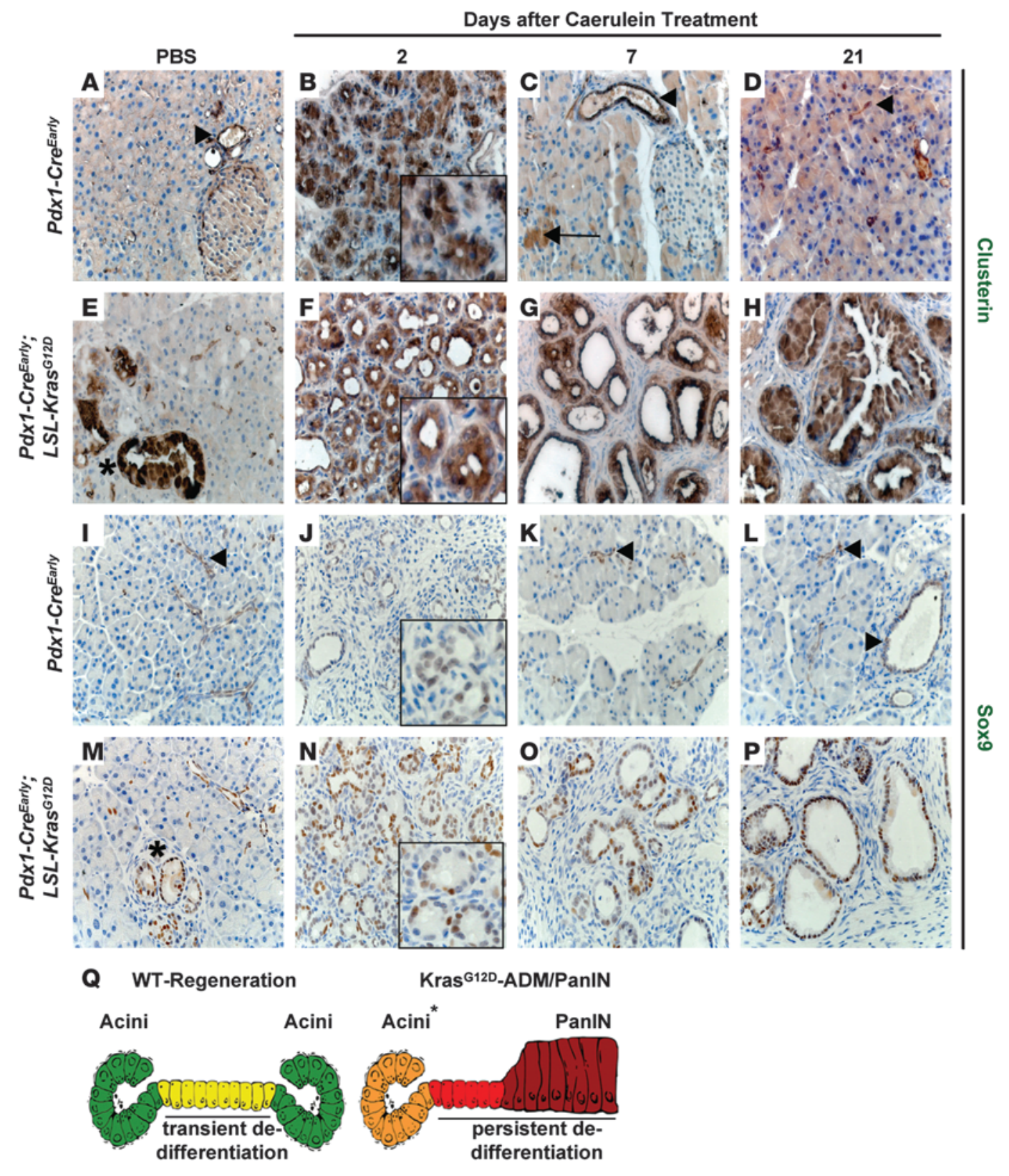

Figure 2

Mutant Kras blocks acinar regeneration in favor of a persistently dedifferentiated state. (A-H) Clusterin and Sox9 (I-P) expression during regeneration and Kras-induced ADM. (A and I) Clusterin expression is limited to some normal ducts in PBS-treated Pdx1-CreEarly mice, while Sox9 is restricted to ducts and centroacinar cells (arrowheads). (E and M) Acini in PBS-treated Pdx1-Cre Early;LSL-Kras ${ }^{G 12 D}$ mice are negative for Clusterin and Sox9, while normal ducts and spontaneous PanINs are positive (asterisks). (B, F, J, and N) Damaged duct-like cells of both genotypes display clusterin- and Sox9-positive cells (insets). (C, D, K, and L) Clusterin and Sox9 are mainly restricted to duct cells (arrowheads) following regeneration in Pdx1-Cre Early pancreata. Rare clusterin-positive cells were observed 7 days following caerulein treatment (arrow, C). (G, $\mathbf{H}, \mathbf{O}$, and $\mathbf{P}$ ) Clusterin and Sox9 remain strongly expressed in ADM and PanINs in Pdx1-CreEarly;LSL-Kras G12D mice. (Q) Schematic of failed regeneration of acini possessing mutant Kras (acini $\left.{ }^{\star}\right)$ versus WT. WT acini transiently dedifferentiate and rapidly regenerate, while acini possessing mutant Kras are sensitized to persistent dedifferentiation and ADM/PanIN formation. Original magnification, $\times 400$ (A-P; insets).

persisted 7 days following caerulein treatment (Figure 1S and Supplemental Figure 1M). Due to the acinar origin of these structures, they may represent persistent TC1-type structures. 21 days following caerulein treatment, we observed frequent doubleCK19/YFP-positive, acinar-derived PanIN lesions (Figure 1T and Supplemental Figure 1N). Therefore, mutant Kras subverts the regenerative capacity of otherwise morphologically normal acini following acute pancreatitis and instead promotes a fixed ductal fate that permits ADM/PanIN formation.

Mutant Kras alters acinar regeneration by maintaining a persistently dedifferentiated state. Since mutant Kras subverts acinar cell fate decisions following damage, we next asked whether mutant Kras affects the 

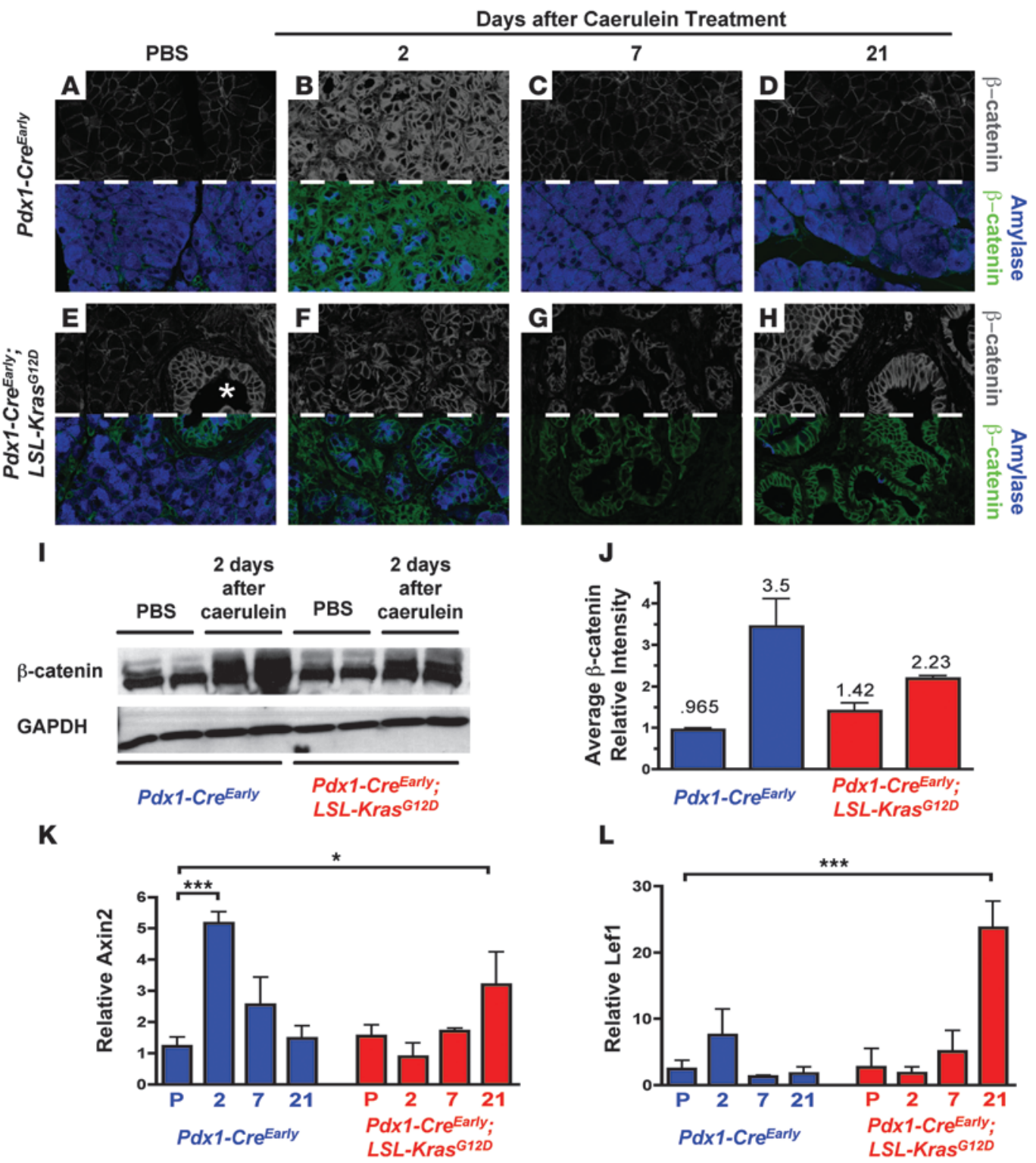

Figure 3

Regeneration-associated reactivation of $\beta$-catenin signaling is inhibited during Kras-induced ductal reprogramming. (A-H) $\beta$-catenin (above dashed line, gray; below dashed line, green) and amylase (above dashed line, not shown; below dashed line, blue) immunofluorescent staining. (A-D) Pdx1-Cre Early mice; (E-H) Pdx1-Cre Early; $L S L-K r a s^{G 12 D}$ mice. Asterisk in E marks spontaneous PanIN lesions. (I and $\left.\mathbf{J}\right)$ Western blot analysis of $\beta$-catenin 2 days following caerulein treatment. Intensity, normalized to GAPDH, is quantified in $\mathbf{J}$ (bars represent mean \pm SD). (K and $\mathbf{L}$ ) Quantitative PCR for $\beta$-catenin target genes during WT acinar regeneration (blue bars) and ADM/PanIN (red bars). Values are relative to PBS-treated $P d x 1$-Cre Early mice and are presented as mean \pm SD $(n=3)$. P, PBS treatment; $2,7,21$ indicate days after caerulein treatment. ${ }^{\star} P<0.05 ;{ }^{* \star *} P<0.001$. Original magnification, $\times 630(\mathbf{A}-\mathbf{H})$.

reactivation of embryonic signaling pathways during normal acinar regeneration. Two days following caerulein treatment, the exocrine compartments in both Pdx1-Cre $e^{\text {Early }}$ and Pdx1-Cre $e^{E a r l y} ; L S L-K r a s^{G 12 D}$ mice displayed reactivation of elements of embryonic development or markers of immature acini, frequently in duct-like structures, including Clusterin (Figure 2, B and F) (15), Sex-determining region Y (SRY) box 9 (Sox9) (Figure 2, J and N) (26), Pdx1 (Supplemental Figure 2, B and F) (14), and the Notch target gene homolog of Drosophila hairy and enhancer of split 1 (Hes1) (Supplemental Figure 2, J and N) (14). This suggests that exocrine cells possessing mutant
Kras assume progenitor-like properties in response to acinar damage similar to that of WT exocrine cells. The adult expression pattern of these embryonic factors before injury was reestablished 7 and 21 days following caerulein administration in Pdx1-Cre Early control mice (Figure 2, C, D, K, and L, and Supplemental Figure 2, C, $\mathrm{D}, \mathrm{K}$, and $\mathrm{L}$ ). In stark contrast, expression of all tested embryonic factors persisted in ductal metaplasia and in PanINs observed in Pdx 1-Cre ${ }^{\text {Early }} ;$ LSL-Kras ${ }^{G 12 D}$ mice (Figure 2, G, H, O, and P), similar to spontaneous PanINs found in age-matched PBS-treated mice (Figure 2, E and M, and Supplemental Figure 2, E and M). 

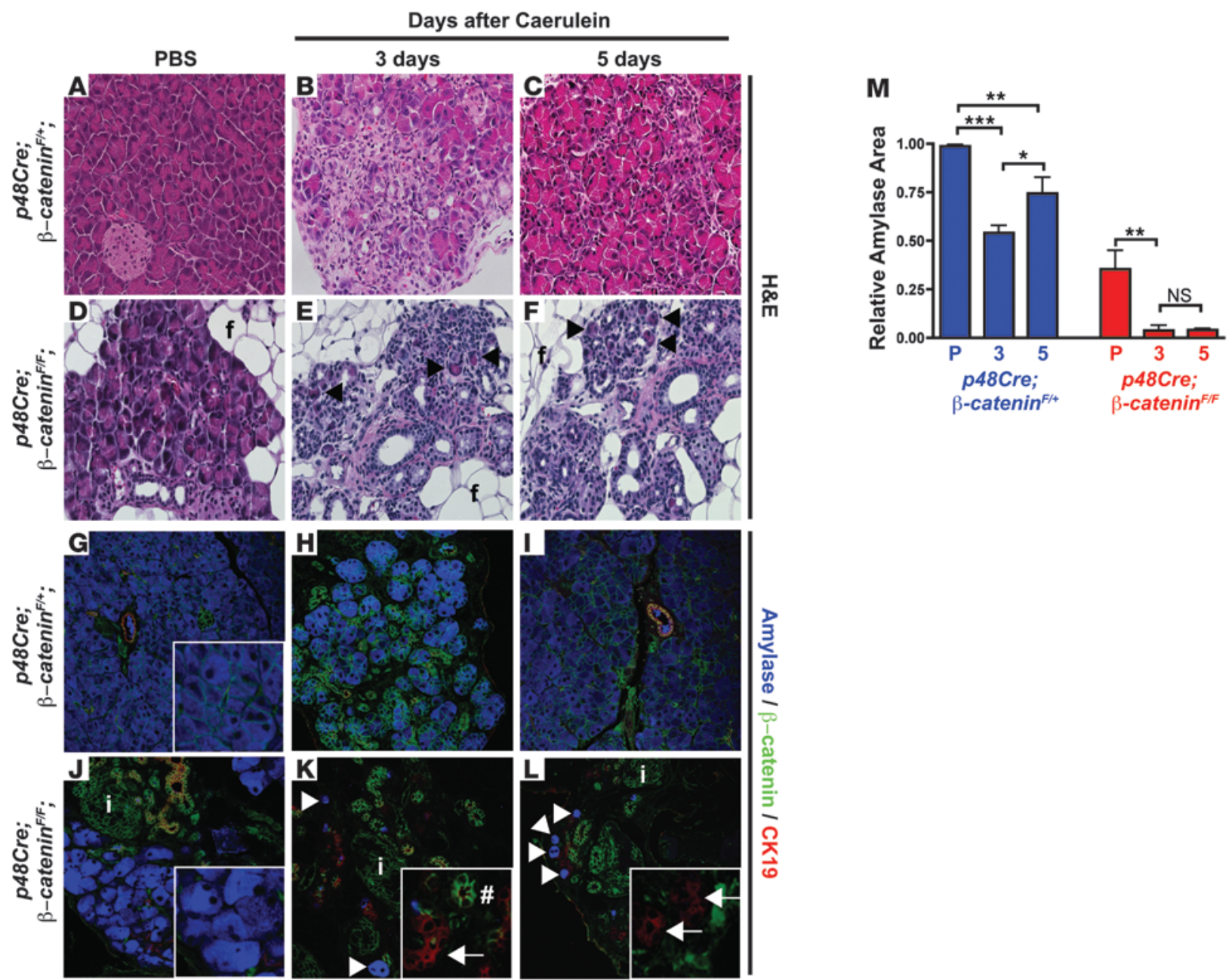

\section{Figure 4}

$\beta$-catenin is required for efficient acinar regeneration. (A-F) H\&E staining of regenerating control $\left(p 48 C r e ; \beta-c a t e n i n^{F /+}\right)$ versus $p 48 C r e ; \beta$-catenin ${ }^{F / F}$ pancreas following caerulein treatment. f, fat accumulation (D-F). Rare acini following caerulein treatment are marked with arrowheads (E and F). (G-L) $\beta$-catenin (green), amylase (blue), and CK19 (red) immunofluorescent labeling. Acini in p48Cre; $\beta$-catenin ${ }^{F / F}$ mice lack membrane $\beta$-catenin staining (compare insets in $\mathbf{G}$ and $\mathbf{J}$ ). Arrowheads mark rare acini ( $\mathbf{K}$ and $\mathbf{L})$. Both $\beta$-catenin-negative (arrows) and -positive (hatch marks) CK19-labeled ducts are observed. i, islets. (M) Quantification of relative amylase area in control (blue bars) and $p 48 C r e ; \beta-c a t e n i n^{F / F}$ (red bars) following caerulein pancreatitis. Bars represent mean \pm SD. P, PBS treatment. 3 and 5 indicate days after caerulein treatment. ${ }^{*} P<0.05$; ${ }^{\star \star} P<0.01$; ${ }^{\star \star \star} P<0.001$. Original magnification, $\times 400$ (A-L, insets).

Next, we directly compared acinar differentiation during regeneration and $\mathrm{ADM} /$ PanIN formation in Elastase-Cre ${ }^{E R T 2} ; R 26 R-E Y F P$ and Elastase-Cre ${ }^{E R T 2}$;LSL-Kras ${ }^{G 12 D}$;R26R-EYFP mice. Consistent with our observations in Pdx1-Cre $e^{E a r l y}$ and $P d x 1-C r e^{E a r l y} ; L S L-K r a s^{G 12 D}$ mice, we observed acinar-derived (YFP-positive) cells in both tamoxifen-stimulated ElastaseCre ${ }^{\text {ERT2}} ;$ R26R-EYFP and Elastase-Cre ${ }^{\text {ERT2}} ;$ LSL-Kras ${ }^{\text {G12D; }}$ R26REYFP mice that expressed Clusterin (Supplemental Figure 3B), Sox9 (Supplemental Figure 3J), and Pdx1 (data not shown) 2 days following caerulein treatment. As expected, strong expression of Clusterin, Sox9, and Pdx1 was absent from acini in ElastaseCre ${ }^{E R T 2} ; R 26 R-E Y F P$ mice when regeneration was complete (Supplemental Figure 3, C, D, $\mathrm{K}$, and $\mathrm{L}$, and data not shown). In contrast, acinar-derived metaplastic ducts and PanINs in Elastase-Cre ${ }^{\text {ERT2}}$;LSL-Kras ${ }^{G 12 D}$;R26R-EYFP mice maintained strong expression of these embryonic factors (Supplemental Figure 3, G, H, O, and P, and data not shown). Therefore, mutant Kras not only diverts regeneration toward a ductal fate, but also promotes a persistently dedifferentiated state (Figure 2Q).
We also asked whether maintenance of a dedifferentiated ductal state corresponded with persistent activation of Kras effector pathways. As assessed by phosphorylation of both p42 and p44 (ERK1/2), which are readouts for the activity of the MAPK pathway downstream of Kras signaling, we found that the pattern of Kras activity matched that of the persistently active embryonic factors. While spontaneous ADM/PanIN in PBS-treated Pdx1Cre $e^{\text {Early }} ; L S L-K r a s^{G 12 D}$ mice displayed phospho-ERK1/2 reactivity, it was mainly absent in morphologically normal acini (Supplemental Figure 4E). However, phospho-ERK1/2 staining was frequently observed in cells assuming a ductal morphology 2 days following caerulein treatment (Supplemental Figure 4F) and was maintained in subsequent metaplastic ducts and PanINs (7 and 21 days following caerulein treatment; Supplemental Figure 4, $\mathrm{G}$ and $\mathrm{H})$. In the regenerating exocrine compartment of control $P d x 1$-Cre ${ }^{\text {Early }}$ mice, phospho-ERK1/2 was observed in a subset of cells 2 days following caerulein treatment (Supplemental Figure 


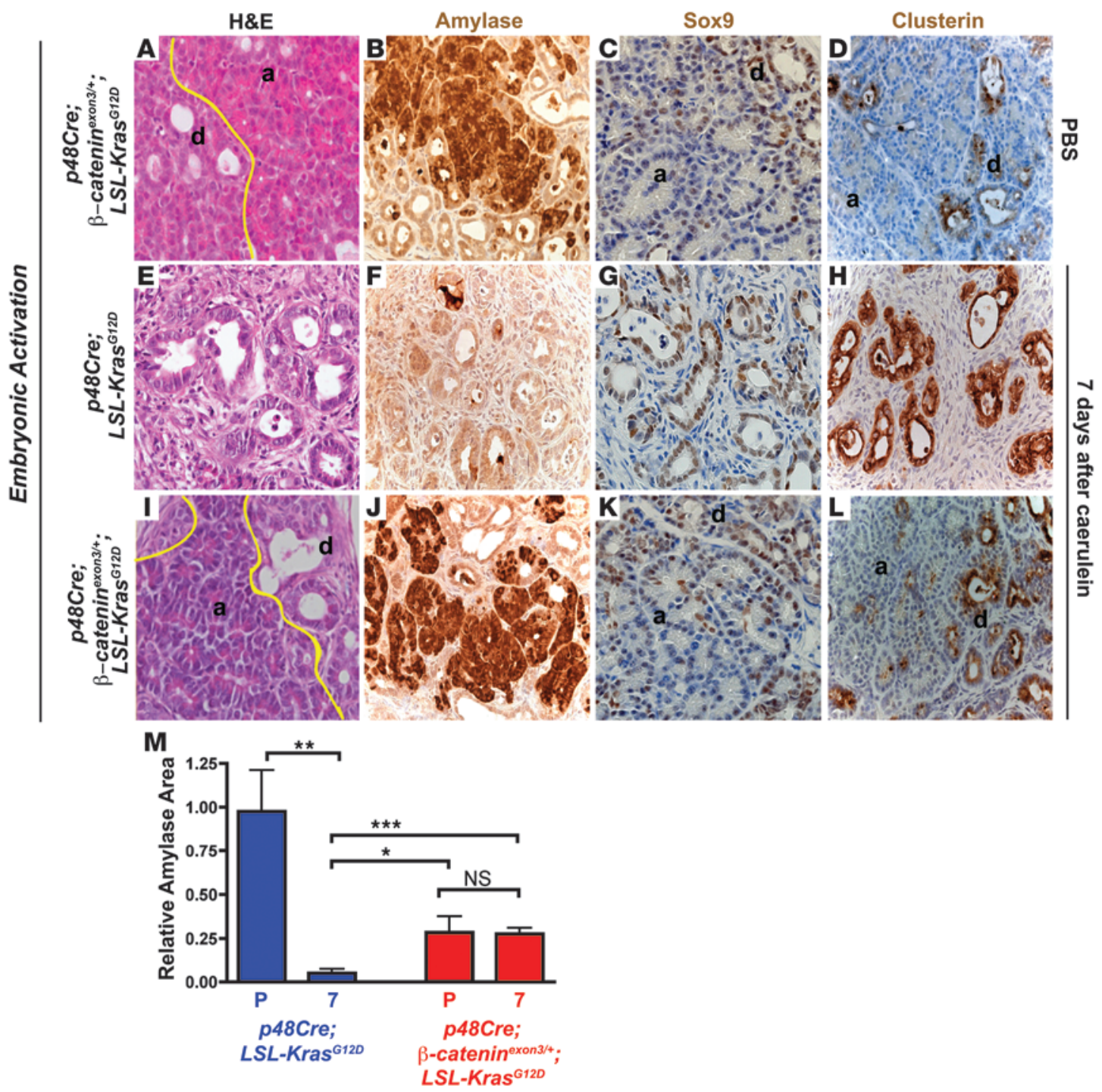

\section{Figure 5}

Stabilized $\beta$-catenin antagonizes Kras-induced ADM. (A-L) Characterization of 6-week-old PBS- and caerulein-treated $p 48 C r e ; \beta$-cateninexon3/+; LSL-Kras ${ }^{G 12 D}$ mice and caerulein-treated p48Cre;LSL-Kras ${ }^{G 12 D}$ mice. (A, E, and I) H\&E staining. Yellow lines separate areas of cells with acinar morphology (a), and ductal morphology (d). (B, F, and J) Amylase staining. (C, G, and K) Sox9 staining. (D, H, and L) Clusterin staining. (M) Quantification of relative amylase area 7 days following caerulein in $p 48 \mathrm{Cre} ; \beta$-catenin ${ }^{E x o n} 3 /+; L S L-K r a S^{G 12 D}$ (red bars) and control $p 48 C$ re; LSL$\operatorname{Kras}^{G 12 D}$ (blue bars) mice. Bars represent mean \pm SD. 7 indicates days after caerulein treatment. ${ }^{*} P<0.05 ;{ }^{* *} P<0.01 ;{ }^{* * *} P<0.001$. Original magnification, $\times 400(\mathbf{A}-\mathbf{L})$.

4B), but was not found in acini in PBS-treated animals (Supplemental Figure 4A) or in acini following regeneration (Supplemental Figure 4, C and D). In summary, these data suggest that the regenerative environment produced by acute pancreatic damage not only promotes cell-fate changes that permit Kras-induced $\mathrm{ADM} /$ PanIN formation, but may also provide an environment that permits sustained Kras effector function.

Regeneration-associated $\beta$-catenin signaling is inbibited during Kras-induced acinar-to-ductal reprogramming. Since ductal metaplasia is synchronized and widespread in caerulein-treated Pdx1-Cre ${ }^{\text {Early }} ;$ LSL-Kras ${ }^{G 12 D}$ mice, this model provides an opportunity to identify key signaling pathways that are inappropriately modulated in the presence of mutant Kras to prevent regeneration and promote ADM/PanIN. One candidate pathway is $\beta$-catenin signaling. $\beta$-catenin is the prime transcriptional activator of canonical Wnt signaling and has been implicated in regeneration of many organs, including the liver, lung, and limbs (reviewed in ref. 27). During pancreatic development, $\beta$-catenin is critical for acinar specification $(28,29)$ and has been implicated in endocrine development (30). Furthermore, $\beta$-catenin has been shown to accumulate in regenerating WT acini following caerulein pancreatitis (14), although a role for $\beta$-catenin signaling activity has not been investigated during pancreas regeneration. Thus, we compared $\beta$-catenin accumulation and signaling during acinar regeneration and Kras-induced ADM/PanIN formation.

In PBS-treated $P d x 1$-Cre $e^{E a r l y}$ mice, weak $\beta$-catenin signal was apparent at the membrane of acinar cells and ducts (Figure $3 \mathrm{~A}$ and data not shown). While spontaneous PanINs in PBS-treated Pdx1$C r e^{E a r l y} ; L S L-K r a s^{G 12 D}$ mice frequently displayed strong membranous 


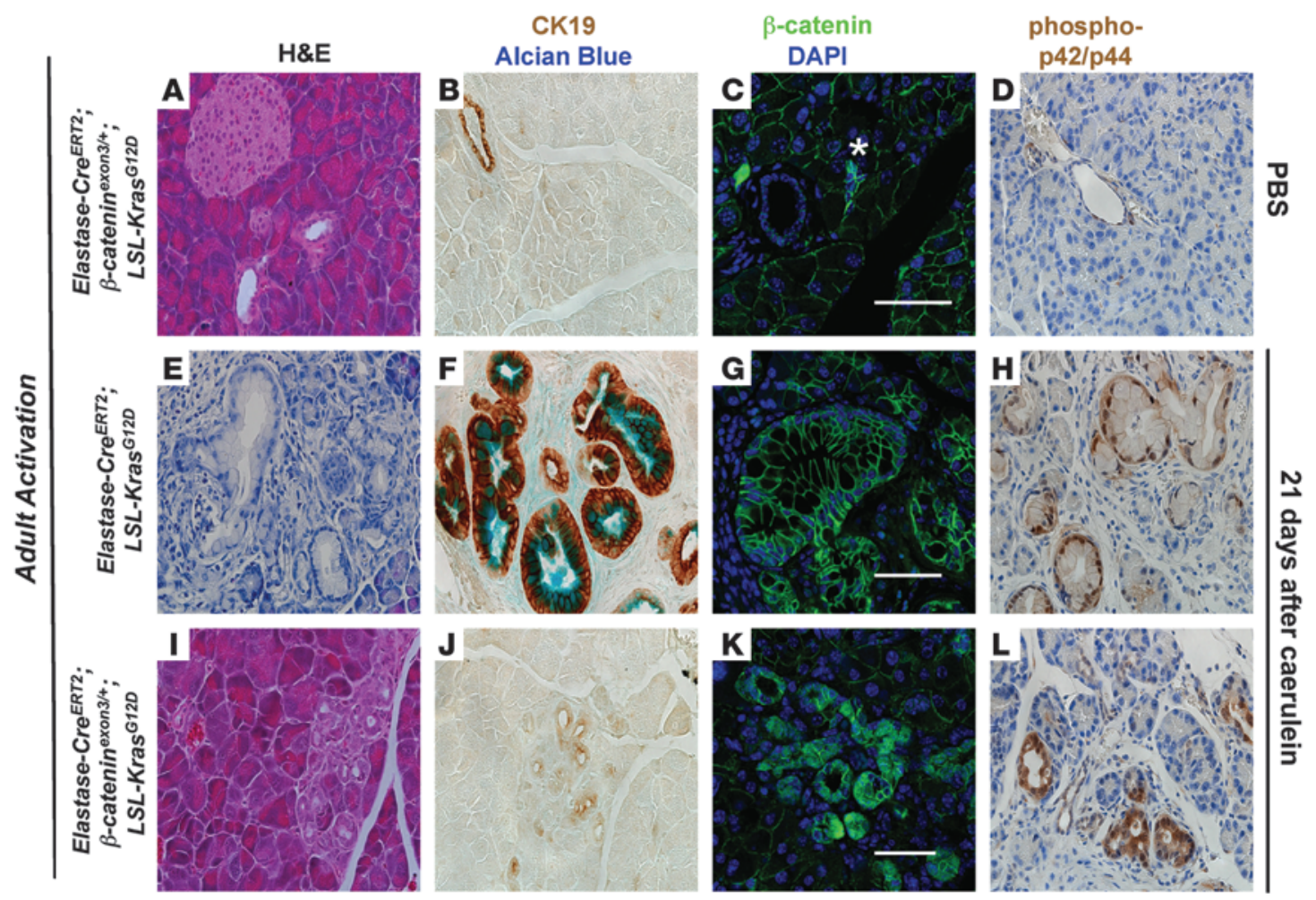

\section{Figure 6}

$\beta$-catenin signaling inhibits Kras-induced reprogramming of acini into PanINs. (A, E, and I) H\&E staining of tamoxifen-stimulated, caerulein- or PBS-treated ElaCre ${ }^{E R T} ; \beta$-cateninexon3/+;LSL-Kras ${ }^{G 12 D}$ or ElaCre ${ }^{E R T} ; L S L-K r a s^{G 12 D}$ mice. (B, F, and J) CK19/Alcian blue staining. Note that normal ducts in PBS-treated ElaCre ${ }^{E R T} ; \beta$-cateninexon3/+;LSL-Kras G12D mice are strongly CK19 positive but Alcian blue negative (B). (C, G, and K) $\beta$-catenin (green), DAPI (blue) immunofluorescent labeling. $\beta$-catenin accumulation is rare in PBS-treated ElaCre ${ }^{E R T} ; \beta$-cateninexon3/+;LSL-Kras ${ }^{G 12 D}$ mice (asterisk, $\mathbf{C}$ ). Nuclear localization is noted by cyan overlap of green/blue channels (K). (D, H, and $\mathbf{L}$ ) phospho-p42/p44 staining. Original magnification, $\times 400(\mathbf{A}, \mathbf{B}, \mathbf{D}-\mathbf{F}, \mathbf{H}-\mathbf{J}$, and $\mathbf{L})$. Scale bars: $50 \mu \mathrm{m}(\mathbf{C}, \mathbf{G}$, and $\mathbf{K})$.

and cytoplasmic $\beta$-catenin staining, morphologically normal acini presented with weak membranous labeling comparable to that of PBS-treated Pdx1-Cre Early acini (Figure 3E). Two days after caerulein treatment, cells in the regenerating exocrine compartment of $P d x 1$ $C r e^{E a r l y}$ mice displayed accumulation of $\beta$-catenin, predominately at the cell periphery and in the cytoplasm (Figure 3B). Like other embryonic factors, $\beta$-catenin distribution reverted to the pattern of PBS-treated mice at 7 and 21 days after treatment (Figure 3, C and D). In contrast to other reactivated developmental signaling factors, the damaged exocrine compartment in Pdx1-Cre ${ }^{\text {Early }} ; \mathrm{LSL}$ $\operatorname{Kras}^{G 12 D}$ mice displayed weaker overall $\beta$-catenin levels as well as less frequent cytoplasmic accumulation 2 days following caerulein treatment compared with cells in similarly treated $P d x 1-C r e^{E a r l} y$ control mice (Figure $3 \mathrm{~F}$ ). Western blot for $\beta$-catenin protein confirmed these immunostaining results (Figure 3I; quantification of Western blot results shown in Figure 3J). In metaplastic ducts 7 days following treatment, overall $\beta$-catenin accumulation was still weaker than that observed during the regenerative phase in $P d x 1-C r e^{E a r l y}$ mice (Figure 3G). Only after 3 weeks did $\beta$-catenin frequently accumulate in the cytoplasm of PanIN lesions (Figure 3H).

Quantitative PCR analysis of the canonical Wnt signaling/ $\beta$-catenin target genes Axin2 (a negative regulator of $\beta$-catenin signaling) and lymphoid enhancer binding factor 1 (Lef1) revealed a pattern of $\beta$-catenin transcriptional activation corresponding with the observed pattern of $\beta$-catenin accumulation. In $P d x 1$ -
Cre $e^{\text {Early }}$ mice, expression of both genes increased 2 days following caerulein treatment and returned to PBS-treated levels by 21 days (Figure 3, K and L). We detected no increase in Axin2 and Lef1 expression during ADM 2 and 7 days following caerulein treatment of Pdx1-Cre $e^{E a r l y} ; L S L-K r a s^{G 12 D}$ mice, while both genes were significantly upregulated when PanINs predominated after 21 days (Figure 3, K and L). Therefore, a distinction between transiently dedifferentiated, regenerating WT acini and acini possessing mutant Kras undergoing the initial stages of ductal reprogramming is the inability of the latter to normally reactivate Wnt/ $\beta$-catenin signaling. We also tested to determine whether previously reported regeneration-associated accumulation of E-cadherin $(14,31)$, which binds to $\beta$-catenin at adherens junctions, was similarly inhibited during Kras-induced ADM. We observed accumulation of E-cadherin 2 days after caerulein treatment in both $P d x 1$ Cre ${ }^{\text {Early }}$ and Pdx1-Cre ${ }^{\text {Early }}$;LSL-Kras ${ }^{G 12 D}$ mice (Supplemental Figure $5, \mathrm{~B}$ and $\mathrm{F}$ ), suggesting that Kras-induced ADM is not associated with a gross decrease in E-cadherin accumulation.

$\beta$-catenin is required for efficient acinar cell regeneration. Next we aimed to determine whether $\beta$-catenin inhibition is an important component of Kras-driven ADM and PanIN formation. First, we asked whether $\beta$-catenin plays a functional role in acinar regeneration. To address this question, we set out to subject acinar cells lacking $\beta$-catenin to caerulein pancreatitis and gauge their regenerative ability. $\beta$-catenin is required for acinar development 


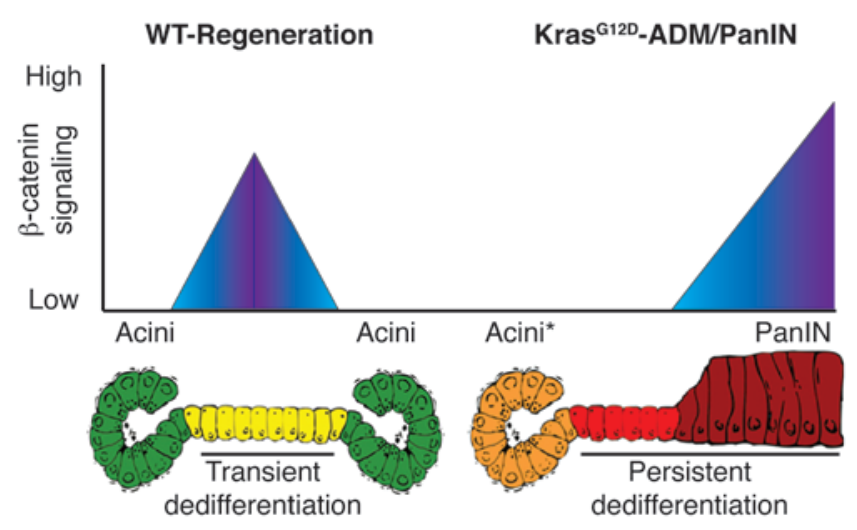

Figure 7

$\beta$-catenin acts as a gatekeeper of Kras-induced reprogramming of acini into ductal PanINs. Pattern of $\beta$-catenin signaling activity during acinar regeneration versus Kras-induced ADM/PanIN. $\beta$-catenin levels are kept below a critical threshold during the initiation of Kras-induced ductal reprogramming but increase as PanIN lesions form. Therefore, $\beta$-catenin antagonizes specification of a ductal state capable of forming PanINs, but likely contributes to PanIN progression and tumor growth.

(28-30), but not adult acinar cell viability or differentiation (28) as shown by conditional inactivation of a Cre-dependent, floxed $\beta$-catenin allele $\left(\beta\right.$-catenin $\left.{ }^{F / F}\right)$. We have previously demonstrated that Cre recombinase driven by the pancreas-specific transcription factor 1a (Ptf1a) ( $p 48)$ promoter induces recombination in both acini and a subset of ductal progenitors (32). Although its expression occurs during the early stages of pancreas development, we have found that using it to conditionally manipulate the $\beta$-catenin pathway avoids developmental phenotypes induced by earlier acting Cre drivers, such as the $P d x 1-C r e^{\text {Early }}$ strain (22). We generated $p 48 \mathrm{Cre} ; \beta$-catenin ${ }^{F / F}$ mice, which were viable and born at the expected Mendelian ratio, and tested to determine whether they possessed $\beta$-catenin-negative acinar cells at 6 weeks of age. Similar to previous results investigating $\beta$-catenin deletion during pancreas development, the number of acinar cells was severely reduced in $p 48 \mathrm{Cre} ; \beta$-catenin ${ }^{F / F}$ mice (representing $~ 35 \%$ of the area of controls; Figure 4M), and pancreata displayed accumulation of fat (Figure 4D). However, the remaining pancreas was composed of $\beta$-catenin-null, amylase-positive acinar cells (Figure 4J) along with a mixture of $\beta$-catenin-negative and -positive ducts and islets. Therefore, although $\mathrm{p} 48$-positive acinar progenitors develop far less efficiently without $\beta$-catenin than WT counterparts, $p 48 \mathrm{Cre} ; \beta$-catenin ${ }^{F / F}$ mice were tractable for testing the ability of $\beta$-catenin-null acinar cells to regenerate.

Next, we compared acinar regeneration following caerulein pancreatitis in control $p 48 \mathrm{Cre} ; \beta$-catenin ${ }^{F /+}$ and $p 48 \mathrm{Cre} ; \beta$-catenin ${ }^{F / F}$ mice. We induced pancreatitis using the method of Jensen and colleagues (14) in which mice are subjected to consecutive days of caerulein treatment. We employed this particular regimen, as it has been frequently used to test genetic modifiers of acinar regeneration. Previous studies have shown that in response to this caerulein protocol, acinar cells both assume a duct-like morphology (16) and reactivate embryonic-signaling factors $(14,15)$ similar to those we observed in mice treated with the staggered caerulein regimen that we used in our ADM/PanIN studies (see Figures 1 and 2). Therefore, similar regenerative pathways are likely induced by both caerulein techniques. As expected, 3 days after caerulein treatment,
p48Cre; $\beta$-catenin ${ }^{F /+}$ control mice displayed a significant decrease in acinar area, approximately $50 \%-60 \%$ of PBS-treated control (Figure $4 \mathrm{M}$ ), possessed regenerating acini, including duct-like cells (Figure $4 \mathrm{~B}$ ), and displayed increased $\beta$-catenin accumulation compared with acini in PBS-treated mice (compare Figure 4, G and H). By 5 days following treatment, acinar regeneration in $p 48 \mathrm{Cre} ; \beta$-catenin ${ }^{F /+}$ mice had significantly progressed, as nearly $75 \%$ of acinar area was reestablished (Figure 4M). In contrast, at both 3 and 5 days following caerulein treatment, $p 48 \mathrm{Cre} ; \beta$-catenin ${ }^{F / F}$ mutant mice displayed a persistent decrease in $\beta$-catenin-negative, amylase-positive cells. Quantitative analysis revealed a reduction of greater than $90 \%$ of acinar area when compared with PBS-treated $p 48 \mathrm{Cre} ; \beta$-catenin ${ }^{F /+}$ mice and an approximately 10 -fold reduction (Figure $4 \mathrm{M}$ ) when compared with PBS-treated $p 48 \mathrm{Cre} ; \beta$-catenin ${ }^{F / F}$ mice (Figure 4, $\mathrm{E}, \mathrm{K}, \mathrm{F}$, and $\mathrm{L}$, respectively). The remaining epithelium consisted predominately of both $\beta$-catenin-negative and CK19-positive duct cells as well as $\beta$-catenin-negative and-positive endocrine cells remaining in morphologically distinct islet clusters (Figure 4, K, L, and insets). These data suggest that $\beta$-catenin-deficient acinar cells regenerate far less efficiently than WT acini and support a role for $\beta$-catenin as a key node in acinar regeneration.

Stabilized $\beta$-catenin signaling antagonizes Kras-driven ADM/PanIN. Since $\beta$-catenin is important in acinar regeneration and a block in its reactivation correlates with Kras-induced ductal reprogramming, we asked next whether increased $\beta$-catenin signaling could antagonize the ability of Kras to reprogram acini into ADM/PanIN. To answer this question, we used a gain-of-function strategy to enforce $\beta$-catenin signaling in acini possessing mutant Kras.

To induce stabilized $\beta$-catenin in acini coexpressing mutant Kras, we utilized a Cre-induced conditional transgenic allele of $\beta$-catenin that lacks exon 3 ( $\beta$-catenin $\left.{ }^{\text {exon } 3}\right)$, a region required for proteasomal turnover (33), in combination with the LSL-Kras ${ }^{G 12 D}$ allele. We have previously shown that 1 copy of this allele increases $\beta$-catenin signaling in pancreatic epithelium $(22,32)$. We analyzed the effect of simultaneous Kras and $\beta$-catenin activation on ductal reprogramming in mice in which both pathways were activated in the developing exocrine compartment and specifically in adult acini. For studies of embryonic activation, we again used the $p 48 \mathrm{Cre}$ driver to activate the conditional $\beta$-catenin allele rather than the Pdx1-Cre ${ }^{E a r l y}$ allele employed earlier in this study to avoid previously reported developmental defects and perinatal lethality (22).

First, we compared pancreata of PBS-injected, 6-week-old p48Cre;LSL-Kras ${ }^{G 12 D}$ and compound $p 48 \mathrm{Cre} ; \beta$-catenin ${ }^{\text {exon } 3 /+} ; L S L$ Kras $^{G 12 D}$ mice. As expected, $p 48 C r e ; L S L-K r a s^{G 12 D}$ pancreas displayed morphologically normal acinar cells with few PanIN lesions (Supplemental Figure 6A).p48Cre; $\beta$-catenin ${ }^{\text {exon } 3 /+} ;$ $L S L-K r a s^{G 12 D}$ mice presented with a mixture of abnormal duct cells and amylase-positive cells with acinar morphology, representing approximately $25 \%$ of the acinar area of $p 48 \mathrm{Cre}$; LSL-Kras ${ }^{G 12 D}$ animals (Figure 5, A, B, and $\mathrm{M})$. Strikingly, PanIN lesions were not observed under these conditions. Therefore, even at this early time point and in the absence of a damaging insult that accelerates Kras-induced PanIN formation, the propensity of mutant Kras to drive PanIN development is inhibited by stabilized $\beta$-catenin. In support of this notion, we have previously shown that $p 48 \mathrm{Cre} ; \beta$-catenin ${ }^{\text {exon } 3 /+} ;$;SL-Kras ${ }^{G 12 D}$ mice develop tumors resembling human intraductal tubular tumors (ITT) as they age but show evidence of neither PanIN lesions nor PDA (32).

Next, we compared acinar replacement in p48Cre;LSL-Kras ${ }^{G 12 D}$ and compound $p 48 \mathrm{Cre} ; \beta$-catenin ${ }^{\text {exon } 3 /+} ; L S L-K r a s^{G 12 D}$ mice 7 days following caerulein treatment. As expected, caerulein treatment in 
p48Cre;LSL-Kras ${ }^{G 12 D}$ mice resulted in a significant replacement of acinar cells with metaplastic ducts, leading to a decrease of amylase area to less than $10 \%$ of that in PBS-treated mice (Figure 5, F and $\mathrm{M})$. As observed in Pdx1-Cre $e^{E a r l y} ; L S L-K r a s^{G 12 D}$ mice, metaplastic ducts (Figure 5E) strongly expressed embryonic factors (Sox9, Figure 5G; Clusterin5H, Hes1, Supplemental Figure 7A) and displayed phospho-ERK1/2 labeling (Supplemental Figure 7B). In stark contrast, cells with acinar morphology were maintained with a similar distribution 7 days following caerulein treatment in p48Cre; $\beta$-catenin ${ }^{\text {exon } 3 /+} ; L S L-K r a s^{G 12 D}$ animals as compared with mice treated with PBS (Figure 5, J and M). Even 2 days after treatment, at the height of caerulein-induced dedifferentiation, the distribution of acini and ductal cells in $p 48 \mathrm{Cre} ; \beta$-catenin ${ }^{\text {exon } 3 /+} ; L S L-\mathrm{Kras}^{G 12 D}$ mice was similar to that observed in PBS-treated mice (Supplemental Figure 6, G and H). Furthermore, Sox9 (Figure 5K), Clusterin (Figure 5L), Hes1 (Supplemental Figure 7E), and phospho-ERK1/2 (Supplemental Figure 7F) were rarely present in cells with acinar morphology in $p 48 \mathrm{Cre} ; \beta$-catenin ${ }_{\text {exon } 3 /{ }^{+}} ;$LSL-Kras ${ }^{G 12 D}$ mice (Figure 5, $\mathrm{I}$ and J) regardless of caerulein treatment. Immunofluorescence for $\beta$-catenin displayed widespread accumulation in all epithelial cell types in $448 \mathrm{Cre} ; \beta$-catenin ${ }^{\text {exon } 3 /+} ;$ $L S L-$ Kras $^{G 12 D}$ pancreas compared with that of $p 48 \mathrm{Cre} ; L S L-\mathrm{Kras}^{G 12 D}$ mice, confirming transgenic $\beta$-catenin stabilization (compare Supplemental Figure 7, D and $\mathrm{H})$. $\beta$-catenin activation was also confirmed by quantitative PCR for target genes Axin 2 and Lef1, which displayed significant increase in $p 48 \mathrm{Cre} ; \beta$-catenin ${ }^{\text {exon } 3 /+} ; \mathrm{LSL}-\mathrm{Kras}{ }^{G 12 D}$ versus $p 48 \mathrm{Cre} ; \mathrm{LSL}$ Kras ${ }^{G 12 D}$ pancreas (data not shown). Taken together, these data suggest that when activated simultaneously during pancreatic development, $\beta$-catenin signaling antagonizes the normal course of Kras-induced acinar reprogramming.

We have previously established that $p 48 \mathrm{Cre} ; \beta$-catenin ${ }^{\text {exon } 3 /+} ; L S L$ $\operatorname{Kras}^{G 12 D}$ mice do not develop PanIN lesions (32). However, in this model, Cre activation is not limited to acini and mice develop significant abnormalities, including fibrosis, that may exert non-cell autonomous effects on acinar reprogramming. Therefore, to more rigorously interrogate the ability of $\beta$-catenin signaling to antagonize Kras-induced ADM/PanIN originating from adult acini, we compared tamoxifen-stimulated, caerulein-treated Elastase-Cre ${ }^{E R T 2} ;$ LSL-Kras ${ }^{G 12 D}$ and Elastase-Cre ${ }^{E R T 2} ; \beta$-cateninexon3/+; $L S L-$ ras $^{G 12 D}$ mice 2 and 3 weeks following pancreatitis. Unlike p48Cre; $\beta$-catenin ${ }^{\text {exon } 3 /+} ;$ LSL-Kras ${ }^{G 12 D}$ mice, tamoxifen-stimulated Elastase-Cre ${ }^{\text {ERT2 }} ; \beta$-catenin ${ }^{\text {exon } 3 /+} ;$ LSL-Kras ${ }^{G 12 D}$ animals showed no gross structural defects when treated either with PBS or with caerulein (Figure 6A). Furthermore, cellular defects, in the form of abnormal ductal structures, were rare in PBS-treated Elastase-Cre ${ }^{E R T 2}$; $\beta$-catenin ${ }^{\text {exon } 3 /+} ; L S L-K_{r a s}^{G 12 D}$ mice (such ducts were observed in 1 of 7 mice 4 to 5 weeks after tamoxifen treatment). This frequency matched that of spontaneous PanINs observed in similarly treated Elastase-Cre ${ }^{E R T 2}$;LSL-Kras ${ }^{G 12 D}$ mice (Figure 1 and Supplemental Figure 1). Therefore, without caerulein treatment, the effects of activating Kras alone or both Kras and $\beta$-catenin simultaneously were minimal, presenting an opportunity to determine the ability of stabilized $\beta$-catenin signaling to affect Kras-induced ADM/PanIN in the absence of preexisting structural defects.

Two and 3 weeks following caerulein treatment, all Elastase$\mathrm{Cre}^{\text {ERT2}} ; \mathrm{LSL}-\mathrm{Kras}^{\mathrm{G} 12 D}$ mice tested $(n=4, n=3)$ displayed frequent CK19, Alcian blue-positive PanINs (Figure 6, E and F). However, PanIN formation was blocked in Elastase-Cre $e^{E R T 2} ; \beta$-catenin ${ }^{\text {exon } 3 /+} ; L S L$ Kras ${ }^{G 12 D}$ mice, as we observed no structures that morphologically resembled PanINs at either time point $(n=4, n=3)$. While we did observe CK19-positive abnormal duct structures in all caeruleintreated Elastase-Cre ${ }^{\text {ERT2 }} ; \beta$-catenin ${ }^{\text {exon } 3 /+} ; L S L-K r a S^{G 12 D}$ mice, cells that made up the ducts maintained a cuboidal rather than columnar morphology and generally possessed weak CK19 expression (Figure 6, I and J). Furthermore, in contrast to PanINs observed in Elastase-Cre ${ }^{E R T 2}$; LSL-Kras ${ }^{G 12 D}$ mice, Alcian blue staining was exceedingly infrequent in these structures $(2$ of 7 mice possessed 1 to 2 structures that displayed patchy or weakly Alcian blue-positive cells) (Figure 6J and data not shown). Interestingly, we found that distinct patterns of $\beta$-catenin accumulation correlated with the development of PanINs or abnormal duct structures. PanINs in Elastase-Cre ${ }^{E R T 2} ; L S L-K r^{G}{ }^{G 12 D}$ mice possessed strong membranous and cytoplasmic $\beta$-catenin accumulation, while $\beta$-catenin was frequently localized to the nucleus in the abnormal ducts observed in Elastase-Cre ${ }^{\text {ERT2}} ; \beta$-catenin ${ }^{\text {exon3/+*}}$;SSL-Kras ${ }^{G 12 D}$ mice (compare Figure 6 , G and $\mathrm{K}$ ). However, both structures displayed phospho-ERK1/2 staining (Figure 6, H and L). Therefore, while a Kras effector pathway is active in both cell types, abnormal ducts in ElaCre ${ }^{E R T 2} ; L S L$ Kras ${ }^{G 12 D} ; \beta$-catenin ${ }^{\text {exon } 3 /+}$ mice are likely subjected to a higher level of $\beta$-catenin signaling than cells that make up PanINs. Taken together, these data suggest that while uncontrolled $\beta$-catenin and Kras signaling can change acinar fate, levels of $\beta$-catenin signaling that exceed a critical threshold block the ability of Kras to reprogram acini into a ductal PanIN lineage.

\section{Discussion}

Regeneration is a key process in maintaining tissue homeostasis. Interestingly, regenerative processes often involve reactivation of developmental signaling pathways that may contribute to tumorigenesis when aberrantly active in adult tissue. This balance between regeneration and neoplasia appears to be pertinent in the pancreas as both acute pancreatitis, an insult that produces a regenerative response in pancreatic acini $(14,15,16)$, and chronic pancreatitis accelerate Kras-driven ADM/PanIN and PDA in mice (refs. 11 and 5, respectively). These studies support clinical observations suggesting a link between chronic pancreatitis and PDA in humans $(12,13)$. However, how Kras coopts the permissive environment provided by pancreatitis to initiate reprogramming of acini into ductal PanINs is unclear. Here, we show that one route by which Kras drives ADM and PanIN formation is by subverting acinar regeneration. Our results demonstrate that acini possessing an activating mutation in Kras are biologically distinct from normal acini. As schematized in Figure 2Q, while possessing normal morphology and expressing markers of acinar differentiation, acinar cells carrying constitutively active Kras cannot activate a WT regenerative program. Instead, these cells undergo ductal reprogramming when damaged, a state characterized by persistent, rather than transient, progenitor factor expression, which permits the development of PanINs that are molecularly similar to spontaneous lesions. As progenitor factor expression is maintained from the outset of ADM/PanIN following damage, our data suggest that dedifferentiation may be a key rate-limiting step in initiating Kras transformation of acini into PanIN precursors.

While mutant Kras is sufficient to reprogram acini into ductal PanINs, this process occurs gradually and stochastically. Tissue damage (i.e., pancreatitis; present study and refs. 5, 11), inflammation induced by metabolic stress (34), and additional oncogene activation such as activated Gli (35), Notch (3), and TGF- $\alpha$ signaling (36), significantly accelerate ADM/PanIN. Therefore, expression of mutant Kras is not optimally efficient to reprogram acini 
into PanINs without additional molecular events. During Krasinduced $\mathrm{ADM} / \mathrm{PanIN}$ following damage, we found not only persistent activation of regeneration-associated embryonic signaling factors, but also that the activity of the MAPK Kras effector pathway was widely and persistently maintained. This is in contrast to morphologically normal acini in age-matched, control-treated mice in which MAPK activity was infrequently observed. These data suggest that this dedifferentiated state is not only plastic for ADM and PanIN formation but may permit increased signaling output by Kras effectors. Recently, Ji and colleagues (37) reported a relationship between increasing levels of acinar Kras activity and the development of chronic pancreatitis or ADM/PanIN with the capacity to give rise to PDA. Interestingly, Duan and colleagues (38) have shown that cholecystokinin, which caerulein mimics functionally, can increase Ras activity. Therefore, although we did not directly assay Kras activity levels in our studies, caerulein treatment itself may be involved in breaching a threshold of Ras activity critical to initiating ADM/PanIN. Even so, the question remains of whether dedifferentiation is a consequence of Kras activity or is required for Kras activation to occur. Combining the ability to titrate Kras activity in acini with genetic techniques that target the reactivated embryonic signaling pathways observed during ADM/PanIN may make an epistatic relationship between differentiation and Kras signaling more clear. Another critical future direction is to determine what molecular roadblocks prevent Kras from becoming fully active in unstressed acini and how they are subverted under conditions of damage or spontaneously to allow ADM/PanIN. Identification of such factors might supply diagnostic or therapeutic targets that are aimed at the specification of PDA precursors.

The accelerated and synchronous ADM induced in response to acute pancreatic damage in our models of embryonic Kras activation allowed us to isolate $\beta$-catenin as an essential player in acinar regeneration and Kras-mediated reprogramming of PDA precursors. $\beta$-catenin is critical for acinar development (28-30) but does not seem to be required for the viability of unstressed adult acini (28). We find that $\beta$-catenin is essential for acinar regeneration. $\beta$-catenin has significant effects on both gene expression and on cell-to-cell adhesion as a part of complexes at the membrane. Since target genes are upregulated during acinar regeneration, our data suggest that $\beta$-catenin-dependent transcription may be involved in establishing a regenerative program. Further experiments detailing how specific $\beta$-catenin target genes direct regeneration and whether $\beta$-catenin-dependent adhesion is critical to the process are key to better understanding this aspect of acinar plasticity.

Since $\beta$-catenin is key for acinar development, we expected it to share the expression pattern of other reactivated embryonic factors and remain persistently reactivated during Kras-induced ADM. Surprisingly, we found that while $\beta$-catenin signaling was activated during normal acinar regeneration, such transient activation was not observed during early stages of Kras-induced ADM/PanIN. Instead, we found that $\beta$-catenin signaling did not increase until PanIN lesions began to predominate in the pancreatic epithelium. Our gain-of-function studies suggest that this balance between active Kras signaling and low levels of $\beta$-catenin signaling is key to specifying a duct-like lineage capable of forming PanINs. In response to damage, acini expressing mutant Kras and stabilized $\beta$-catenin throughout development do not undergo ADM as readily as acini expressing mutant Kras alone. Cells maintaining acinar morphology rarely express the reactivated elements of embryonic development or phospho-MAPK as seen persistently in acini undergoing
Kras-induced ADM/PanIN. Also, stabilized $\beta$-catenin signaling prevents Kras-induced reprogramming of adult acini into PanIN lesions. Therefore, high levels of $\beta$-catenin signaling are incompatible with the molecular environment that enables Kras to specify a ductal lineage with the capacity to develop into PDA. Interestingly, this interaction may be similar to the antagonism of $\beta$-catenin target genes by Hras signaling that is observed in the zonation of periportal versus pericentral hepatocytes in the liver $(39,40)$, although the mechanism of inhibition in this context is unclear. Understanding how $\beta$-catenin levels and signaling are controlled during the initiation of ADM/PanIN, whether Kras directly inhibits $\beta$-catenin, and which $\beta$-catenin targets must be blocked for Kras to take advantage of an otherwise permissive differentiation state also may provide new insight into the development of PDA precursors as well as an important developmental signaling interaction.

Our findings elucidate seemingly contradictory data regarding Kras and $\beta$-catenin signaling in PDA initiation and maintenance. It is well established that $\beta$-catenin and Kras signaling synergize to drive tumorigenesis in other organs, including the colon and prostate $(41,42)$. Our group has previously shown that while Kras and $\beta$-catenin do ultimately synergize to induce tumor formation in aged $p 48 \mathrm{Cre} ; \beta$-catenin ${ }^{\text {exon } 3 /+} ; \mathrm{LSL}$-Kras ${ }^{G 12 D}$ mice, the ITT-like disease caused by simultaneous Kras activation and $\beta$-catenin stabilization throughout acinar cell development is molecularly and morphologically distinct from the Kras-induced PanIN-PDA sequelae (32). These results suggest that PDA cannot form in the context of simultaneous, constitutive activation of Kras and $\beta$-catenin signaling. However, $\beta$-catenin signaling is elevated in human PDA and in PanINs and PDA of Kras-driven mouse models $(43,44)$. Indeed, our results support this notion since we observed Wnt target gene upregulation in Kras mice 21 days after caerulein treatment, a time point in which PanIN formation is widespread. Furthermore, $\beta$-catenin knockdown in human PDA cell lines possessing activating Kras mutations compromises cell growth (43). Our study presents a possible explanation for the temporal dissonance in the relationship between Kras and $\beta$-catenin in PDA. As schematized in Figure 7, we propose that $\beta$-catenin signaling is a gatekeeper for Kras-induced reprogramming of acini and must be kept below a critical threshold in order for Kras to drive cells into forming a metaplastic lineage with the potential to develop into PanIN/PDA. Once this state is achieved, cells fated for PDA can evolve a requirement for $\beta$-catenin signaling, possibly through stimulating effects supplied by other signaling pathways, including Hedgehog/Gli signaling (43).

Recently, concomitant activation of Notch and Kras in adult acinar cells has been shown to greatly accelerate ADM/PanIN formation (3). Notch signaling plays critical roles in pancreatic development (45) and must be inactivated in pancreatic progenitors in order to permit differentiation of exocrine and endocrine cells (46). We show that the Notch target gene Hes1, normally reactivated transiently during acinar regeneration, is persistently upregulated during ADM/PanIN formation. Siveke and colleagues have shown that Notch signaling is not only important in exocrine regeneration but that Notch also inhibits $\beta$-catenin in acinar cells (15). Taken together, this may suggest that persistently active Notch signaling may be part of the mechanism preventing $\beta$-catenin reactivation during Kras reprogramming of acini into the PanIN lineage.

In conclusion, this study shows that acinar regeneration provides a permissive environment for Kras to induce early events in PDA initiation, promoting ADM/PanIN. Reactivation of $\beta$-catenin signaling, required for efficient acinar regeneration, is blocked dur- 
ing Kras-induced ductal reprogramming, and unless a critical level of $\beta$-catenin activity is maintained, Kras-induced acinar-to-ductal reprogramming into a PanIN lineage is inhibited. Therefore, our study underlines the likelihood that not only must mutations be acquired in a specific sequence in order to develop PDA, but levels of developmental signaling pathways must be tightly regulated to alter normal cellular plasticity and drive neoplastic cell fates.

\section{Methods}

Mouse lines. Experimental animals were generated by crossing Pdx1-Cre Early (gift of Doug Melton, Harvard University, Cambridge, Massachusetts, USA) (47); Pdx1-Cre Late (gift of Pedro Herrera, University of Geneva Medical School, Geneva, Switzerland); p48Cre (gift of Chris Wright, Vanderbilt University, Nashville, Tennessee, USA) (48); or Elastase-Cre ERT2 mice (gift of Doris Stoffers, University of Pennsylvania, Philadelphia, Pennsylvania, USA) with $L S L-K r a s^{G 12 D}$ (gift of Dave Tuveson, Cancer Research UK Cambridge Research Institute, Cambridge, United Kingdom), $\beta$-catenin $n^{F / F}$ (49),

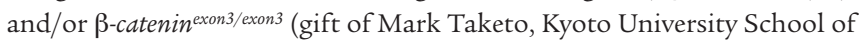
Medicine, Kyoto, Japan), and R26R-EYFP (24). All mouse experiments were performed under the approval of the UCSF Institutional Care and Use of Animals Committee (IACUC). Littermate heterozygote conditional or Creonly animals were used as controls.

Tamoxifen treatment. Cre activity in Elastase-Cre ${ }^{E R T 2} ; R 26 R-E Y F P$, Elastase$C_{r}{ }^{E R T 2} ; L S L-K r a s^{G 12 D} ; R 26 R-E Y F P$, Elastase-Cre ${ }^{E R T 2} ; L S L-K r a s^{G 12 D}$ and Elastase$\mathrm{Cre}^{\text {ERT2}} ; \beta$-catenin ${ }^{\text {exon } 3 /+} ; \mathrm{LSL}-\mathrm{Kras}^{\mathrm{G} 12 \mathrm{D}}$ mice was induced in 4 -week-old mice by 5 consecutive daily treatments of $1 \mathrm{mg}$ of tamoxifen (Sigma-Aldrich) dissolved in corn oil (to a concentration of $10 \mathrm{mg} / \mathrm{ml}$; Sigma-Aldrich).

Caerulein treatment. Acute pancreatitis was induced at 6 weeks of age in Pdx1-Cre ${ }^{\text {Early }} ;$ LSL-Kras ${ }^{G 12 D}$, Pdx1-Cre ${ }^{L a t e} ;$ LSL-Kras ${ }^{G 12 D}$, p48Cre; LSL-Kras ${ }^{G 12 D}$, p48Cre; $\beta$-catenin ${ }^{\text {exon } 3}, p 48 \mathrm{Cre} ; L S L-K r a s^{G 12 D} ; \beta$-catenin ${ }^{\text {exon } 3 l+}$, and tamoxifen induced Elastase-Cre ${ }^{E R T 2} ; R 26 R-E Y F P$, Elastase-Cre ${ }^{E R T 2} ; L S L-K r a s^{G 12 D} ; R 26 R-$ EYFP, Elastase-Cre ${ }^{E R T 2} ; L S L-K r a s^{G 12 D}$, and Elastase-Cre ${ }^{E R T 2} ; \beta$-catenin ${ }^{\text {exon } 3 /+} ; L S L-$ $\mathrm{Kras}^{G 12 D}$ mice by 2 sets of 6 hourly i.p. caerulein injections $(50 \mu \mathrm{g} / \mathrm{kg}$; SigmaAldrich) on alternating days separated by 24 hours (50). This protocol is referred to as the "staggered" protocol in the text. For the regeneration study in $p 48 \mathrm{Cre} ; \beta$-catenin ${ }^{F / F}$ mice, pancreatitis was induced as described by Jensen and colleagues (14), 1 by 8 hourly caerulein injections $(2 \mu \mathrm{g} /$ injection) on 2 consecutive days. This protocol is referred to as the "consecutive" protocol in the text. Mouse weight ranged from 22 to 25 grams. For both protocols, the final day of caerulein injection was considered day 0 .

Immunohistochemistry and immunofluorescence. Pancreata were fixed overnight in zinc-containing neutral-buffered formalin (Anatech Ltd.), embedded in paraffin, cut into 5 - $\mu \mathrm{m}$-thick sections, and placed on Superfrost Plus slides (Fisher Scientific). Sections were subjected to H\&E, immunohistochemical, and immunofluorescent staining as described (22, $35)$. The following primary antibodies were used: rabbit anti-amylase (1:300; Sigma-Aldrich), rat anti-CK19 (TROMAIII, 1:200 dilution; developed by Rolf Kemler [Max-Planck Institute of Immunobiology, Freiburg, Germany] and obtained from the Hybridoma Bank at the University of Iowa, Iowa City, Iowa, USA), guinea pig anti-Pdx1 (1:1000; gift from Michael German, UCSF), rabbit anti-Hes1 (1:500 dilution; gift from Tetsuo Sudo, Toray Industries Inc., Kamakura, Japan), goat anti-clusterin (1:200; Santa Cruz Biotechnology Inc.), rabbit anti-Sox9 (1:1000; Chemicon), mouse anti$\beta$-catenin (1:200; BD), chicken anti-GFP (1:200; Abcam), and anti phosphop42/p44 (1:200; Cell Signaling Technologies). For immunohistochemistry, biotinylated anti-rabbit (Vector Labs), and anti-goat and anti-rat (Jackson ImmunoResearch Laboratories Inc.) antibodies were used as secondary antibodies at a 1:200 dilution. 3-3'-Diaminobenzidine tetrahydrochloride (Vector Labs) was used as a chromogen. Bright-field images were acquired using a Zeiss Axio Imager D1 scope. For immunofluorescence, Alexa Fluor
555 donkey anti-goat, Alexa Fluor 488 donkey anti-rabbit, Alexa Fluor 488 goat anti-chicken, Alexa Fluor 633 goat anti-mouse, Alexa Fluor 488 goat anti-rabbit, Alexa Fluor 633 goat anti-rat, and Alexa Fluor 555 goat antirat (Molecular Probes; Invitrogen) were used as secondary antibodies at a 1:200 dilution. Confocal images were collected on a Leica SP2 microscope at consistent gain and offset settings.

Western blotting. Immunoblotting was performed by homogenizing tissue samples in RIPA buffer, electrophoresing on 10\% SDS-PAGE gels, incubating with primary antibodies overnight at $4^{\circ} \mathrm{C}$, and detecting primary antibodies using HRP-conjugated secondary antibodies (1:5000 dilution; Santa Cruz Biotechnology Inc.) and ECL (Amersham Biosciences) as described (35). The primary antibodies used were anti-mouse $\beta$-catenin $(1: 1000$; BD) and antirabbit GAPDH (1:1000, Santa Cruz Biotechnology Inc.). Western blots were quantified by calculating an integrated density value (IDV) for each band using Adobe Photoshop CS2 and normalizing to the IDV of GAPDH.

Evaluation of amylase area. To calculate relative amylase area in the $p 48 \mathrm{Cre} ; \beta$-catenin ${ }^{F / F}$ regeneration experiment, 6 random, nonoverlapping, $\times 400$ fluorescent images, separated by a depth of $100 \mu \mathrm{M}$, were collected from 3 mice per condition. For each image, Adobe Photoshop CS2 was used to calculate positive amylase pixel number, which was normalized to number of DAPI-positive nuclei (scored by the "analyze particle" in NIH ImageJ; http://rsbweb.nih.gov/ij/). To quantify relative amylase area in the p48Cre;LSL-Kras ${ }^{G 12 D} ; \beta$-catenin ${ }^{3 /+}$ acinar replacement experiment, 8 random, nonoverlapping, $\times 100$ IHC images, 2 each at consecutive tissue level, separated by a depth of $100 \mu \mathrm{M}$, were collected from 3 mice per condition. For each image, Adobe Photoshop CS2 was used to calculate positive amylase pixel number, which was normalized to pancreas tissue area.

Quantitative PCR. Pancreas tissue was preserved in RNALater (Ambion) overnight at $4{ }^{\circ} \mathrm{C}$ and stored at $-80^{\circ} \mathrm{C}$. Total RNA was extracted by tissue dissociation in TRIzOL reagent (Invitrogen) and purification of chloroform extracted aqueous phase using RNeasy columns (QIAGEN). On-column DNAse treatment was performed according to manufacturer's instructions. RNA quality was audited using an Agilent Bioanalyzer and the RNA Nano Chip Kit (Agilent Technologies). cDNA was synthesized from $1 \mu \mathrm{g}$ of total RNA using Superscript II Reverse Transcriptase (Invitrogen). Taqman QPCR was performed using inventoried probes for mouse Axin2 (Mm01265783_m1; Applied Biosystems) and Lef1 (Mm00550265_m1, Applied Biosystems). Expression levels were normalized using a custom primer/probe set for Gapdh (provided by the Genome Analysis Core at the UCSF Helen Diller Family Comprehensive Cancer Center; sequences listed in Supplemental Table 1).

Statistics. Comparison of means was performed using unpaired Student's $t$ tests, calculated using Prism for Macintosh, version 4. Statistical significance was assumed when $P<0.05 ; P$ values below 0.05 are noted in figure legends. Results are represented as mean $\pm \mathrm{SD}$ where noted. All mouse tissue data represent at least 3 mice unless otherwise noted and represent at least ten $5-\mu \mathrm{M}$ sections unless noted.

\section{Acknowledgments}

The authors would like to thank Renee VanderLaan and Limor Landsman for critical reading of the manuscript and Cecilia Austin for histology assistance. Also, we thank the following for providing us with the indicated mouse lines: Douglas Melton, Pdx1-Cre ${ }^{\text {Early }}$; Pedro. Herrera, Pdx1-Cre ${ }^{\text {Late }}$; C. Wright, p48Cre; D. Stoffers, Elastase-Cre ${ }^{E R T 2}$; D. Tuveson, LSL-Kras ${ }^{G 12 D}$; and M. Taketo, $\beta$-catenin ${ }^{\text {exon3 }}$. Work in M. Hebrok's laboratory is supported by a grant from the Pancreatic Cancer Action Network (PanCAN) and the NIH (CA112537). S. Wang is supported by the NIH under Ruth L. Kirschstein National Research Service Award F32 from the National Cancer Institute and the American College of Surgeons 
Resident Research Scholarship. Imaging experiments were supported by resources from the UCSF Diabetes and Endocrinology Research Center (DERC).

Received for publication June 1, 2009, and accepted in revised form November 18, 2009.

Address correspondence to: Matthias Hebrok, Diabetes Center, Department of Medicine, HSW1119, 513 Parnassus Avenue, UCSF,
San Francisco, California 94143, USA. Phone: (415) 514-0820; Fax: (415) 564-5813: E-mail: mhebrok@diabetes.ucsf.edu.

David A. Cano's present address is: Instituto de Biomedicina de Sevilla, Servicio de Endocrinología y Nutrición, Hospitales Universitarios Virgen del Rocío, Sevilla, Spain.

Shigeki Sekine's present address is: Pathology Division, National Cancer Center Research Institute, Tokyo, Japan.
1. Jemal A, et al. Cancer statistics, 2008. CA Cancer J Clin. 2008;58(2):71-96.

2. Feldmann G, Beaty R, Hruban RH, Maitra A. Molecular genetics of pancreatic intraepithelial neoplasia. J Hepatobiliary Pancreat Surg. 2007;14(3):224-232.

3. De La O JP, et al. Notch and Kras reprogram pancreatic acinar cells to ductal intraepithelial neoplasia. Proc Natl Acad Sci U S A. 2008;105(48):18907-18912.

4. Habbe N, et al. Spontaneous induction of murine pancreatic intraepithelial neoplasia ( $\mathrm{mPanIN}$ ) by acinar cell targeting of oncogenic Kras in adult mice. Proc Natl Acad Sci U S A. 2008;105(48):18913-18918.

5. Guerra C, et al. Chronic pancreatitis is essential for induction of pancreatic ductal adenocarcinoma by K-Ras oncogenes in adult mice. Cancer Cell. 2007;11(3):291-302.

6. Zhu L, Shi G, Schmidt CM, Hruban RH, Konieczny SF. Acinar cells contribute to the molecular heterogeneity of pancreatic intraepithelial neoplasia. Am J Pathol. 2007;171(1):263-273.

7. Shi G, et al. Loss of the acinar-restricted transcription factor Mist1 accelerates Kras-induced pancreatic intraepithelial neoplasia. Gastroenterology. 2009;136(4):1368-1378.

8. Almoguera C, Shibata D, Forrester K, Martin J, Arnheim N, Perucho M. Most human carcinomas of the exocrine pancreas contain mutant c-K-ras genes. Cell. 1988;53(4):549-554.

9. Hingorani SR, et al. Preinvasive and invasive ductal pancreatic cancer and its early detection in the mouse. Cancer Cell. 2003;4(6):437-450.

10. Hingorani SR, et al. Trp53R172H and KrasG12D cooperate to promote chromosomal instability and widely metastatic pancreatic ductal adenocarcinoma in mice. Cancer Cell. 2005;7(5):469-483.

11. Carriere C, Young AL, Gunn JR, Longnecker DS, Korc M. Acute pancreatitis markedly accelerates pancreatic cancer progression in mice expressing oncogenic Kras. Biochem Biophys Res Commun. 2009;382(3):561-565.

12. Lowenfels $A B$, et al. Pancreatitis and the risk of pancreatic cancer. International Pancreatitis Study Group. NEngl J Med. 1993;328(20):1433-1437.

13. Malka D, et al. Risk of pancreatic adenocarcinoma in chronic pancreatitis. Gut. 2002;51(6):849-852.

14. Jensen JN, Cameron E, Garay MV, Starkey TW, Gianani R, Jensen J. Recapitulation of elements of embryonic development in adult mouse pancreatic regeneration. Gastroenterology. 2005;128(3):728-741.

15. Siveke JT, et al. Notch signaling is required for exocrine regeneration after acute pancreatitis. Gastroenterology. 2008;134(2):544-555.

16. Fendrich $\mathrm{V}$, et al. Hedgehog signaling is required for effective regeneration of exocrine pancreas. Gastroenterology. 2008;135(2):621-631.

17. Strobel $O$, et al. In vivo lineage tracing defines the role of acinar-to-ductal transdifferentiation in inflammatory ductal metaplasia. Gastroenterology. 2007;133(6):1999-2009.

18. Means AL, et al. Pancreatic epithelial plasticity mediated by acinar cell transdifferentiation and generation of nestin-positive intermediates. Development. 2005;132(16):3767-3776.

19. Sawey ET, Johnson JA, Crawford HC. Matrix metalloproteinase 7 controls pancreatic acinar cell transdifferentiation by activating the Notch signaling pathway. Proc Natl Acad Sci U S A. 2007;104(49):19327-19332.

20. Miyamoto Y, et al. Notch mediates TGF alphainduced changes in epithelial differentiation during pancreatic tumorigenesis. Cancer Cell. 2003;3(6):565-576.

21. Miyatsuka T, et al. Persistent expression of PDX-1 in the pancreas causes acinar-to-ductal metaplasia through Stat 3 activation. Genes Dev. 2006;20(11):1435-1440.

22. Heiser PW, Lau J, Taketo MM, Herrera PL, Hebrok M. Stabilization of beta-catenin impacts pancreas growth. Development. 2006;133(10):2023-2032.

23. Desai BM, et al. Preexisting pancreatic acinar cells contribute to acinar cell, but not islet beta cell, regeneration. J Clin Invest. 2007;117(4):971-977.

24. Srinivas $S$, et al. Cre reporter strains produced by targeted insertion of EYFP and ECFP into the ROSA26 locus. BMC Dev Biol. 2001;1:4.

25. Tokoro T, Tezel E, Nagasaka T, Kaneko T, Nakao A. Differentiation of acinar cells into acinoductular cells in regenerating rat pancreas. Pancreatology. 2003;3(6):487-496.

26. Yoshida T, et al. Expression patterns of epiplakin1 in pancreas, pancreatic cancer and regenerating pancreas. Genes Cells. 2008;13(7):667-678.

27. Stoick-Cooper CL, Moon RT, Weidinger G. Advances in signaling in vertebrate regeneration as a prelude to regenerative medicine. Genes Dev. 2007;21(11):1292-1315.

28. Murtaugh LC, Law AC, Dor Y, Melton DA. Beta-catenin is essential for pancreatic acinar but not islet development. Development. 2005;132(21):4663-4674

29. Wells JM, et al. Wnt/beta-catenin signaling is required for development of the exocrine pancreas. BMC Dev Biol. 2007;7:4.

30. Dessimoz J, Bonnard C, Huelsken J, Grapin-Botton A. Pancreas-specific deletion of beta-catenin reveals Wnt-dependent and Wnt-independent functions during development. Curr Biol. 2005;15(18):1677-1683.

31. Lerch MM, et al. Dissociation and reassembly of adherens junctions during experimental acute pancreatitis. Gastroenterology. 1997;113(4):1355-1366.

32. Heiser PW, et al. Stabilization of beta-catenin induces pancreas tumor formation. Gastroenterology. 2008;135(4):1288-1300.

33. Kemler R, et al. Stabilization of beta-catenin in the mouse zygote leads to premature epithelialmesenchymal transition in the epiblast. Development. 2004;131(23):5817-5824.

34. Khasawneh J, et al. Inflammation and mitochondrial fatty acid beta-oxidation link obesity to early tumor promotion. Proc Natl Acad Sci U S A. 2009;106(9):3354-3359.
35. Pasca di Magliano M, Sekine S, Ermilov A, Ferris J, Dlugosz AA, Hebrok M. Hedgehog/Ras interactions regulate early stages of pancreatic cancer. Genes Dev. 2006;20(22):3161-3173.

36. Siveke JT, Einwachter H, Sipos B, Lubeseder-Martellato C, Kloppel G, Schmid RM. Concomitant pancreatic activation of $\operatorname{Kras}(\mathrm{G} 12 \mathrm{D})$ and Tgfa results in cystic papillary neoplasms reminiscent of human IPMN. Cancer Cell. 2007;12(3):266-279.

37. Ji B, et al. Ras activity levels control the development of pancreatic diseases. Gastroenterology. 2009;137(3):1072-1082.

38. Duan RD, Zheng CF, Guan KL, Williams JA. Activation of MAP kinase kinase (MEK) and Ras by cholecystokinin in rat pancreatic acini. Am J Physiol. 1995;268(6 Pt 1):G1060-G1065.

39. Hailfinger S, Jaworski M, Braeuning A, Buchmann A, Schwarz M. Zonal gene expression in murine liver: lessons from tumors. Hepatology. 2006;43(3):407-414.

40. Braeuning A, Ittrich C, Kohle C, Buchmann A, Schwarz M. Zonal gene expression in mouse liver resembles expression patterns of Ha-ras and betacatenin mutated hepatomas. Drug Metab Dispos. 2007;35(4):503-507.

41. Janssen KP, et al. APC and oncogenic KRAS are synergistic in enhancing Wnt signaling in intestinal tumor formation and progression. Gastroenterology. 2006;131(4):1096-1109.

42. Pearson HB, Phesse TJ, Clarke AR. K-ras and Wnt signaling synergize to accelerate prostate tumorigenesis in the mouse. Cancer Res. 2009; 69(1):94-101.

43. Pasca di Magliano M, et al. Common activation of canonical wnt signaling in pancreatic adenocarcinoma. PLoS ONE. 2007;2(11):e1155.

44. Wang L, et al. Oncogenic function of ATDC in pancreatic cancer through Wnt pathway activation and beta-catenin stabilization. Cancer Cell. 2009;15(3):207-219.

45. Apelquist A, et al. Notch signalling controls pancreatic cell differentiation. Nature. 1999;400(6747):877-881.

46. Esni F, et al. Notch inhibits Ptf1 function and acinar cell differentiation in developing mouse and zebrafish pancreas. Development. 2004;131(17):4213-4224.

47. Gu G, Dubauskaite J, and Melton DA. Direct evidence for the pancreatic lineage: NGN3 + cells are islet progenitors and are distinct from duct progenitors. Development. 2002;129(10):2447-2457.

48. Kawaguchi Y, et al. The role of the transcriptional regulator Ptf1a in converting intestinal to pancreatic progenitors. Nat Genet. 2002;32(1):128-134.

49. Brault $\mathrm{V}$, et al. Inactivation of the beta-catenin gene by Wnt1-Cre-mediated deletion results in dramatic brain malformation and failure of craniofacial development. Development. 2001;128(8):1253-1264.

50. Nagashio Y, et al. Inhibition of transforming growth factor beta decreases pancreatic fibrosis and protects the pancreas against chronic injury in mice. Lab Invest. 2004;84(12):1610-1618. 